\title{
APPROXIMATION OF HOLOMORPHIC MAPS WITH A LOWER BOUND ON THE RANK
}

\author{
DEJAN KOLARIČ
}

(Communicated by Mei-Chi Shaw)

\begin{abstract}
Let $K$ be a closed polydisc or ball in $\mathbb{C}^{n}$, and let $Y$ be a quasiprojective algebraic manifold which is Zariski locally equivalent to $\mathbb{C}^{p}$, or a complement of an algebraic subvariety of codimension $\geq 2$ in such a manifold. If $r$ is an integer satisfying $(n-r+1)(p-r+1) \geq 2$, then every holomorphic map from a neighborhood of $K$ to $Y$ with rank $\geq r$ at every point of $K$ can be approximated uniformly on $K$ by entire maps $\mathbb{C}^{n} \rightarrow Y$ with rank $\geq r$ at every point of $\mathbb{C}^{n}$.
\end{abstract}

\section{INTRODUCTION}

In this paper we consider the following problem of approximating holomorphic maps with a lower bound on their rank. Let $K$ be a closed polydisc (or a closed ball) in a complex Euclidean space $\mathbb{C}^{n}$, and let $f$ be a holomorphic map from an open neighborhood of $K$ to a complex manifold $Y$ such that $\operatorname{rank}_{z} f:=\operatorname{rank}\left(d f_{z}\right) \geq r$ for all $z \in K$, where $r$ is an integer satisfying $1 \leq r \leq \min \{n, \operatorname{dim} Y\}$. Is it possible to approximate $f$ uniformly on $K$ by entire maps $\tilde{\widetilde{f}}: \mathbb{C}^{n} \rightarrow Y$ satisfying $\operatorname{rank}_{z} \tilde{f} \geq r$ at every point $z \in \mathbb{C}^{n}$ ?

The answer clearly depends on the complex analytic properties of $Y$. If $Y$ is Kobayashi hyperbolic [18], this fails already when $r=1$ and $K$ is a disc in $\mathbb{C}$. More generally, if $Y$ is Eisenman $k$-hyperbolic for some $1 \leq k \leq \operatorname{dim} Y$ [5], then $Y$ admits no holomorphic maps $\mathbb{C}^{n} \rightarrow Y$ of rank $\geq k$, and hence the answer is negative for $r \geq k$. More precise quantitative obstructions to the existence of large polydiscs in complex manifolds were obtained by Kodaira [19].

In the positive direction, Forster proved that holomorphic maps $\mathbb{C}^{n} \rightarrow Y=\mathbb{C}^{p}$ satisfy the jet transversality theorem [6], which gives a positive answer if $r$ is sufficiently small compared to $n$ and $p$ (see Theorem 1.6 below). If $r=n<p$, the above rank condition is satisfied by immersions $\mathbb{C}^{n} \rightarrow \mathbb{C}^{p}$, and in this case an affirmative answer follows from the $h$-principle due to Eliashberg and Gromov [14]. If $n>r=p$, the rank condition is satisfied by submersions, and the approximation result follows from the $h$-principle proved by Forstnerič [9]. The Runge approximation problem for holomorphic immersions $\mathbb{C}^{n} \rightarrow \mathbb{C}^{n}$ in the equidimensional case is still open.

Received by the editors June 9, 2006 and, in revised form, December 5, 2006.

2000 Mathematics Subject Classification. Primary 32E30, 32H02, 32M17, 32Q28.

Key words and phrases. Holomorphic maps, approximation, transversality, algebraic sets.

Work on this paper was supported by ARRS, Republic of Slovenia. 
In this paper we consider maps to certain algebraic manifolds. We shall say that a $p$-dimensional complex manifold is of Class $\mathcal{A}_{0}$ if it is quasi-projective algebraic and is covered by finitely many Zariski open sets biregularly isomorphic to $\mathbb{C}^{p}$. Examples include all complex projective spaces and Grassmannians. Class $\mathcal{A}$ will consist of all algebraic manifolds of the form $Y=\widehat{Y} \backslash A$ where $\widehat{Y}$ is a manifold of class $\mathcal{A}_{0}$ and $A$ is a closed algebraic subvariety of $\widehat{Y}$ of complex codimension at least two. (See Definition 2.1 in $§ 2$.) The following is our main result.

Theorem 1.1. Let $K \subset \mathbb{C}^{n}$ be a closed polydisc, a closed ball, or a product of a (lower-dimensional) closed polydisc and a ball. Let $Y$ be a p-dimensional manifold of Class $\mathcal{A}$. Assume that $1 \leq r \leq \min \{n, p\}$, and that $r<n$ if $n=p$. Every holomorphic map $f$ from a neighborhood of $K$ to $Y$ and satisfying $\operatorname{rank}_{z} f \geq r$ at every point $z \in K$ can be approximated uniformly on $K$ by entire maps $\mathbb{C}^{n} \rightarrow Y$ with rank $\geq r$ at every point of $\mathbb{C}^{n}$.

We wish to emphasize that Theorem 1.1 does not follow from the jet transversality theorem, except for values of $r$ which are small compared to $n$ and $p$; compare with Theorem 1.6 below. The following special case may be of particular interest; the analogous result for submersions (when $n>\operatorname{dim} Y$ ) was proved in [9].

Corollary 1.2. Let $K \subset \mathbb{C}^{n}$ and let $Y$ be as in Theorem 1.1. If $n<\operatorname{dim} Y$, then every holomorphic immersion from a neighborhood of $K$ to $Y$ can be approximated uniformly on $K$ by entire immersions $\mathbb{C}^{n} \rightarrow Y$.

Remark 1.3. (Added in final revision.) It was necessary to put restrictions upon $K$ in Theorem 1.1 and Corollary 1.2 since at the time of writing this paper the author was not aware of a better result about approximation with automorphisms than the one contained in Lemma 3.1. Using recent results [16] of S. Kaliman and F. Kutzschebauch it is however possible to prove the main result of the paper (Theorem 1.1) in the case when $K$ is a polynomially convex set in $\mathbb{C}^{n}$ such that:

(1) There is an open neighborhood $\Omega$ of $K$ and a class $\mathcal{C}^{2}$ map $\Phi:[0,1] \times \Omega \rightarrow$ $\mathbb{C}^{n}$ such that for every $t \in(0,1]$ the map $\Phi_{t}(z)=\Phi(t, z)$ is injective and holomorphic,

(2) for $t \in[0,1]$ the image $\Phi_{t}(K)$ is polynomially convex and a subset of $K$,

(3) $\Phi_{0}(z)=z$ for $z \in K$ and $\Phi_{1}$ is a constant map.

To see this, the reader should observe that a main step in the proof involves using an automorphism to send a large polydisc $Q$ containing $K$ to the complement of algebraic variety $\Sigma_{f}$ while at the same time approximating identity on $K$. The first step in Lemma 3.2 was to replace $f$ with an approximation to get $\operatorname{dim} \Sigma_{f} \leq n-2$. Then using Theorem 5 in [16] with $0<t \leq 1$ we obtain an automorphism $\Psi_{t}$ of $\mathbb{C}^{n}$ fixing $\Sigma_{f}$ and approximating $\Phi_{t}$ uniformly on a neighborhood of $K$. Choosing $t$ sufficiently close to 0 , the image $\Psi_{t}(K)$ is contained in a small ball around the origin. Now work with $f^{\prime}=f \circ \Psi_{t}^{-1}, K^{\prime}=\Psi_{t}(K)$ and observe that $K^{\prime} \subset Q^{\prime} \subset$ $\mathbb{C}^{n} \backslash \Sigma_{f^{\prime}}$ for some polydisc $Q^{\prime}$. Use automorphism $\Phi$ (from Lemma 3.1) to push a polydisc containing $\Psi_{t}(Q)$ into $\mathbb{C}^{n} \backslash \Sigma_{f^{\prime}}$ while approximating identity on $Q^{\prime}$. The map $f \circ \Psi_{t}^{-1} \circ \Phi \circ \Psi_{t}$ approximates $f$ on $K$ and satisfies rank condition on $Q$. This allows us to complete the proof of Theorem 1.1 by the inductive scheme decribed below.

In a special case $K$ can be a convex set. One might ask whether Theorem 1.1 holds whenever $K$ is a polynomially convex set in $\mathbb{C}^{n}$. The following example shows 
that this is not the case: the conclusion fails in general if $K$ is not contractible in the complement of a certain algebraic subvariety of codimension 2 .

Example 1.4. Let $\left(z_{1}, z_{2}, w_{1}, w_{2}\right)$ be the coordinates on $\mathbb{C}^{4}$ and let

$$
\Sigma=\mathbb{C}^{2} \times\{0\}^{2} \times \mathbb{C} \subset \mathbb{C}^{5}, Y=\mathbb{C}^{5} \backslash \Sigma, K=\left\{(z, \bar{z}): z \in \mathbb{C}^{2},|z|=1\right\} .
$$

Then $Y$ is a manifold of class $\mathcal{A}$, and the set $K$ is compact polynomially convex in $\mathbb{C}^{4}$ since it is the intersection of the cylinder $|z|=1$ and the totally real plane $w=\bar{z}$. Note that $K$ is a 3 -sphere which is not contractible in $X=\mathbb{C}^{4} \backslash\left(\mathbb{C}^{2} \times\{0\}^{2}\right)$; indeed, it generates the group $\pi_{3}(X)=H_{3}(X, \mathbb{Z})=\mathbb{Z}$. Let $f$ be the inclusion map $\mathbb{C}^{4} \rightarrow \mathbb{C}^{4} \times\{0\} \subset \mathbb{C}^{5}$. Then $f(K) \subset Y$ and $\operatorname{rank} f=4$ on $K$. However, the map $f$ cannot be approximated on $K$ even by a continuous map $F: \mathbb{C}^{4} \rightarrow Y$ since that would imply contractibility of $K$ in $X$, a contradiction.

Manifolds of Class $\mathcal{A}$ were considered by Gromov under the name Ell-regular manifolds [13, §3.5], and by Forstnerič [11, §2]; in those papers the reader can find many further examples. Both classes are stable with respect to blowing up points. Every such manifold $Y$ enjoys the following properties which will play an important role in our proof:

- holomorphic maps from (neighborhoods of) compact convex sets in $\mathbb{C}^{n}$ to $Y$ can be approximated by regular algebraic maps (morphisms) $\mathbb{C}^{n} \rightarrow Y$ [11, Corollary 1.2];

- algebraic maps from affine algebraic manifolds to $Y$ enjoy a version of the jet transversality theorem (see [11, Sect. 5] and $\S 3$ below).

Example 1.5. Theorem 1.1 fails in general for maps to manifolds of the form $Y=\widehat{Y} \backslash A$ where $\widehat{Y}$ is of Class $\mathcal{A}_{0}$ and $A$ is a complex analytic (but not algebraic) subvariety of codimension at least two, or if $A$ contains a hypersurface component. We recall a few examples to this effect:

(1) In $[20, \S 5]$ Rosay and Rudin constructed discrete sets $D \subset \mathbb{C}^{n}$ such that the only holomorphic map $F: \mathbb{C}^{n} \rightarrow \mathbb{C}^{n}$ with nondegenerate Jacobian $(J F \not \equiv 0)$ and satisfying $F\left(\mathbb{C}^{n} \backslash D\right) \subset \mathbb{C}^{n} \backslash D$ is the identity map $F(z)=z\left(z \in \mathbb{C}^{n}\right)$; thus any holomorphic map $F: \mathbb{C}^{n} \rightarrow \mathbb{C}^{n} \backslash D$ has rank $F<n$ at each point.

(2) In $[7, \S 6]$ a proper holomorphic embedding $F: \mathbb{C}^{m} \hookrightarrow \mathbb{C}^{m+n}$ is constructed for any pair of integers $m, n \in \mathbb{N}$ such that the image of any holomorphic map $G: \mathbb{C}^{n} \rightarrow$ $\mathbb{C}^{m+n}$ satisfying $\operatorname{rank}_{z} G=n$ at some point $z \in \mathbb{C}^{n}$ intersects the submanifold $A=F\left(\mathbb{C}^{m}\right) \subset \mathbb{C}^{m+n}$ infinitely many times. It follows that any entire map $\mathbb{C}^{n} \rightarrow$ $\mathbb{C}^{m+n} \backslash A$ has rank $<n$ at each point.

(3) According to Corollary 2 in [4] the complement $\mathbb{P}_{2} \backslash A$ of a very generic algebraic curve $A$ of degree $d \geq 21$ in the projective plane $\mathbb{P}_{2}$ is hyperbolic, i.e., there exist no nonconstant holomorphic maps $\mathbb{C} \rightarrow \mathbb{P}_{2} \backslash A$, and hence Theorem 1.1 fails for $r=1$.

We now give another result whose main ingredient is the jet transversality theorem for holomorphic maps. In many analytic applications it is important to know the dimension of 'degeneration sets' of a generically chosen holomorphic map $f: X \rightarrow Y$ between a given pair of complex manifolds. Denote by $\mathcal{H}(X, Y)$ the space of holomorphic maps $X \rightarrow Y$ equipped with the compact-open topology. By $J^{k}(X, Y)$ we denote the manifold of all $k$-jets of holomorphic maps $X \rightarrow Y$. Given $f \in \mathcal{H}(X, Y)$ and an integer $r \in \mathbb{N}$ we set

$$
\Sigma_{f, r}=\left\{x \in X: \operatorname{rank}_{x} f<r\right\} .
$$


We shall say that $\operatorname{rank} f \geq r$ on a set $K \subset X$ if $\operatorname{rank}_{x} f \geq r$ for all $x \in K$; if we do not specify $K$, it will be understood that $K=X$.

Following [10] we say that a complex manifold $Y$ satisfies the Convex Approximation Property (CAP) if every holomorphic map from a neighborhood of a compact convex set $K \subset \mathbb{C}^{m}(m \in \mathbb{N})$ to $Y$ can be approximated uniformly on $K$ by entire maps $\mathbb{C}^{m} \rightarrow Y$. By the main result of [10] CAP is equivalent to the classical Oka property. Examples of complex manifolds with CAP include complex Lie groups and complex homogeneous spaces.

Theorem 1.6. Let $X$ be a Stein manifold and let $Y$ be a complex manifold satisfying CAP. Let $\operatorname{dim} X=n$, $\operatorname{dim} Y=p$, and let $r$ be an integer satisfying $r \leq \min (n, p)$. Set $d=(n-r+1)(p-r+1)$.

(1) If $d \leq n$, then the set $\Omega=\left\{f \in \mathcal{H}(X, Y): \operatorname{dim} \Sigma_{f, r}=n-d\right\}$ is open and everywhere dense in $\mathcal{H}(X, Y)$.

(2) If $d>n$, then the set $\Omega^{\prime}=\{f \in \mathcal{H}(X, Y): \operatorname{rank} f \geq r\}$ is open and everywhere dense in $\mathcal{H}(X, Y)$.

Remark 1.7. The examples in 1.5 show that Theorem 1.6 fails in general if we do not assume anything on $Y$. In the proof we shall use the jet transversality theorem for holomorphic maps $X \rightarrow Y$ which holds if $X$ is a Stein manifold and $Y$ satisfies CAP [11, Theorem 1.4] or the Ell 1 property introduced by Gromov (see Definition 2.6 below and [11, Theorem 4.2]).

On the other hand, Kaliman and M. Zaidenberg proved in [17] that every holomorphic mapping $f: X \rightarrow Y$ from a Stein manifold $X$ to any complex manifold $Y$ can be approximated on any compact set $K \subset X$ by holomorphic maps from a neighborhood of $K$ to $Y$ whose $k$-jet extension is transversal to a given analytic subset of the jet manifold $J^{k}(X, Y)$. See also [11, Theorem 4.8]. This gives the following analogue of Theorem 1.6:

Let $X$ be a Stein manifold, and let $Y$ be a complex manifold. Let $n, p, r, d$ be as in Theorem 1.6. Given a compact set $K \subset X$ and a holomorphic map $f: X \rightarrow Y$, there is a holomorphic map $\tilde{f}$ from an open neighborhood of $K$ in $X$ to $Y$ which approximates $f$ on $K$ as close as desired and satisfies

(1) if $d \leq n$, then $\operatorname{dim}_{z} \Sigma_{\tilde{f}, r}=n-d$ for all $z \in K$;

(2) if $d>n$, then $\operatorname{rank} \tilde{f} \geq r$ on $K$.

\section{Preliminaries}

Recall [12] that a projective algebraic set (or variety) is a closed subset of a complex projective space $\mathbb{P}_{n}$ of the form

$$
A=\bigcap_{j=1}^{k}\left\{\left[z_{0}: \ldots: z_{n}\right] \in \mathbb{P}_{n}: p_{j}\left(z_{0}, \ldots, z_{n}\right)=0\right\}
$$

where the $p_{j}$ 's are homogeneous holomorphic polynomials on $\mathbb{C}^{n+1}$. Such $A$ is a closed complex analytic subvariety of $\mathbb{P}_{n}$, and every closed complex analytic subvariety of $\mathbb{P}_{n}$ is of this form by Chow's theorem [3, p. 74]. Occasionally we shall omit the adjective 'projective'. The topology on $\mathbb{P}_{n}$ in which the closed sets are exactly the projective algebraic sets is called the Zariski topology on $\mathbb{P}_{n}$. A quasi-projective algebraic set is a difference $Y \backslash Y^{\prime}$ of two closed algebraic subvarieties $Y, Y^{\prime} \subset \mathbb{P}_{n}$. A 
(quasi-) projective algebraic manifold is a (quasi-) projective algebraic set without singularities.

Let $U \subset \mathbb{P}_{n}$ be a quasi-projective algebraic set. A function $f: U \rightarrow \mathbb{C}$ is called a regular function if

$$
f(Z)=f\left(z_{0}, \ldots, z_{n}\right)=P\left(z_{0}, \ldots, z_{n}\right) / Q\left(z_{0}, \ldots, z_{n}\right), \quad Z \in U,
$$

where $P$ and $Q$ are homogeneous polynomials on $\mathbb{C}^{n+1}$ of the same degree and $Q(Z) \neq 0$ for all $Z \in U$. A continuous map $F: U \rightarrow \mathbb{P}_{N}$ is a regular map if its components with respect to any affine chart $\mathbb{C}^{N} \subset \mathbb{P}_{N}$ are regular functions on $U \cap F^{-1}\left(\mathbb{C}^{N}\right)$. If $U^{\prime} \subset \mathbb{P}_{N}$ is another quasi-projective algebraic set, then a bijective map $F: U \rightarrow U^{\prime}$ is a biregular isomorphism if both $F$ and $F^{-1}$ are regular maps.

Definition 2.1 (Class $\mathcal{A}$ manifolds). Let $Y$ be a quasi-projective algebraic manifold.

(i) $Y$ is of Class $\mathcal{A}_{0}$ if it is covered by finitely many Zariski open sets biregularly isomorphic to $\mathbb{C}^{m}, m=\operatorname{dim} Y$.

(ii) $Y$ is of Class $\mathcal{A}$ if $Y=\widehat{Y} \backslash A$, where $\widehat{Y}$ is a manifold of class $\mathcal{A}_{0}$ and $A$ is an algebraic set in $\widehat{Y}$ with complex codimension at least two.

Manifolds of class $\mathcal{A}$ were used by Gromov under the name Ell-regular manifolds $[13, \S 3.5]$; our terminology conforms to the one by Forstnerič [11].

Example 2.2. Complex affine and projective space, as well as complex Grassmannians, are manifolds of Class $\mathcal{A}_{0}$. Further examples are the rational surfaces, i.e., complex surfaces birationally equivalent to $\mathbb{P}_{2}\left[2\right.$, p. 244]. Apart from $\mathbb{P}_{2}$ these include the Hirzebruch surfaces $\Sigma_{n}, n \in \mathbb{Z}_{+}$.

We recall a few relevant notions regarding transversality of mappings.

Definition 2.3. Let $f: X \rightarrow Y$ be a smooth map of manifolds and let $B$ be a smooth submanifold of $Y$. We say that $f$ is transverse to $B$ at a point $x \in X$, denoted by $f \pitchfork_{x} B$, if either (i) $f(x) \notin B$, or (ii) $f(x) \in B$ and $T_{f(x)} Y=T_{f(x)} B+$ $d f_{x}\left(T_{x} X\right)$. If $f \pitchfork_{x} B$ for all $x \in X$, we say that $f$ is transverse to $B$, and denote it by $f \pitchfork B$.

For latter application we state a couple of known transverality lemmas. The first one provides a lot of transversal maps to choose from a transversal family of maps; a proof consists of a reduction to Sard's theorem and can be found in [1] or [23].

Lemma 2.4 (Transversality Lemma). Let $X, Y, P$ be complex manifolds, let $B$ be a complex submanifold of $Y$, and let $\Phi: X \times P \rightarrow Y$ be a holomorphic map such that $\Phi \pitchfork B$. Let $f_{t}(z):=\Phi(z, t)$. Then $\left\{t \in P: f_{t} \pitchfork B\right\}$ is dense in $B$.

The following lemma, together with Sard's theorem, implies a jet version of the Transversality Lemma for holomorphic maps. See [11, Lemma 4.5] or [17]; here we supply some additional details to the proof. Recall that $J^{k}(X, Y)$ denotes the complex manifold of all $k$-jets of holomorphic maps $X \rightarrow Y$ between a pair of complex manifolds.

Lemma 2.5. Let $X$ be a Stein manifold of dimension $r$, embedded as a closed complex submanifold of $\mathbb{C}^{n}$, let $Y$ be a complex manifold of dimension $p$, and let $F: X \times \mathbb{C}^{N} \rightarrow Y$ be a holomorphic map such that for every $x \in X$ the map $F(x, \cdot): \mathbb{C}^{N} \rightarrow Y$ is a submersion at $0 \in \mathbb{C}^{N}$. Let $W$ denote the vector space of all 
holomorphic polynomial maps $P: \mathbb{C}^{n} \rightarrow \mathbb{C}^{N}$ of degree $\leq k$. For each $P \in W$ set $F_{P}(x)=F(x, P(x)), x \in X$. Then the map $H: X \times W \rightarrow J^{k}(X, Y)$, defined by $H(x, P)=j_{x}^{k}\left(F_{P}\right)$, is a submersion in a neighborhood of $X \times\{0\}$ in $X \times \mathbb{C}^{N}$.

Proof. We need to prove that $H$ is a submersion at points $\left(x_{0}, 0\right) \in X \times W$. Set $F\left(x_{0}, 0\right)=y_{0}$. Choose a neighborhood $U$ of $x_{0}$ in $\mathbb{C}^{n}$, a neighborhood $V$ of $y_{0}$ in $Y$ and a neighborhood $E$ of 0 in $W$ such that $\Phi(X \cap U)=\Phi(U) \cap\left(\mathbb{C}^{r} \times\{0\}^{n-r}\right)=U^{\prime}$ for biholomorphic maps $\Phi: U \rightarrow \Phi(U) \subset \mathbb{C}^{n}, \Psi: V \rightarrow \Psi(V)=V^{\prime} \subset \mathbb{C}^{p}$ and such that $F((X \cap U) \times E) \subset V$. We can consider $U^{\prime}$ to be an open subset of $\mathbb{C}^{r}$. The map $\Psi_{1}: J^{k}(X \cap U, V) \rightarrow J^{k}\left(U^{\prime}, V^{\prime}\right)$ sending $j_{x}^{k} f \in J^{k}(X \cap U, V)$ to $j_{\Phi(x)}^{k}\left(\Psi \circ f \circ \Phi^{-1}\right)$ is well defined and biholomorphic. Let $H^{\prime}=\Psi_{1} \circ H \circ\left(\left.\Phi\right|_{U \cap X} \times\right.$ id $)^{-1}$. Then $H^{\prime}: U^{\prime} \times E \rightarrow J^{k}\left(U^{\prime}, V^{\prime}\right)$ with $H^{\prime}(\Phi(x), P)=j_{\Phi(x)}^{k} F^{\prime}(\cdot, P(\cdot))$, where $F^{\prime}=\Psi \circ F \circ\left(\left.\Phi\right|_{X \cap U} \times \mathrm{id}\right)^{-1}$. It is enough to show that $H^{\prime}$ is a submersion at $(\Phi(x), 0)$. Therefore we can assume that $X=\mathbb{C}^{r} \subset \mathbb{C}^{n}$ and also $Y=\mathbb{C}^{p}$.

Given $x_{0} \in \mathbb{C}^{r}$ we denote by $W_{x_{0}}$ the set of all polynomials $P \in W$ such that $P\left(x_{0}\right)=0$. For a fixed $x \in \mathbb{C}^{r} \subset \mathbb{C}^{n}$ the map $W_{x} \rightarrow \mathbb{C}^{M(r, N, k)}, P \mapsto \partial_{x}^{k} P(x)$, is a submersion. Here $J^{k}\left(\mathbb{C}^{r}, \mathbb{C}^{N}\right)=\mathbb{C}^{r} \times \mathbb{C}^{N} \times \mathbb{C}^{M(r, N, k)}$ for some $M(r, N, k) \in \mathbb{N}$ and $\partial_{x}^{k} P(x)$ denotes all partial derivatives of $P$ of order less than or equal to $k$ without the 0 -th derivative. For every multiindex $I=\left(i_{1}, \ldots, i_{r}\right)$ we can write

$$
\partial_{x}^{I}(F(x, P(x)))=\sum_{j=1}^{n} \frac{\partial F}{\partial t_{j}}(x, P(x)) \partial_{x}^{I} P_{j}(x)+R(x) .
$$

Here $R$ contains derivatives of $P$ of order lower than $|I|$ and derivatives of $F$. For a fixed $x=x_{0}$ we get

$$
\left.\sum_{j=1}^{n} \frac{\partial F}{\partial t_{j}}\left(x_{0}, 0\right) \partial_{x}^{I}\right|_{x=x_{0}} P_{j}(x)+R\left(x_{0}\right)=\left.\left.\partial_{t}\right|_{t=0} F\left(x_{0}, \cdot\right) \cdot \partial_{x}^{I}\right|_{x=x_{0}} P(\cdot)+R\left(x_{0}\right),
$$

where $R\left(x_{0}\right)$ depends linearly on the components of $j_{x_{0}}^{(|I|-1)} P(x)$. We also see that $H\left(x_{0}, P\right)$ is a block-wise lower triangular linear map in the base $\left\{\partial_{x_{0}}^{J} P(x),|J| \leq k\right\}$ of $\mathbb{C}^{M(r, N, k)}$. Since $\left.\partial_{t}\right|_{t=0} F\left(x_{0}, \cdot\right)$ is surjective, the map $P \mapsto j_{x_{0}}^{k}\left(F_{P}\right)$ from $W_{x_{0}}$ to $\mathbb{C}^{N(r, p, k)}$ is a submersion, and hence $H$ is a submersion at $\left(x_{0}, 0\right)$.

Definition 2.6. Let $X$ and $Y$ be complex manifolds. Holomorphic maps $X \rightarrow Y$ satisfy Condition $E l_{1}$ if for every map $f \in \mathcal{H}(X, Y)$ there is a holomorphic map $H: X \times \mathbb{C}^{N} \rightarrow Y$ for some $N \in \mathbb{N}$, satisfying

(1) $H(x, 0)=f(x)$ for all $x \in X$, and

(2) the map $H(x, \cdot): \mathbb{C}^{N} \rightarrow Y$ is a submersion at $0 \in \mathbb{C}^{N}$ for every $x \in X$.

The Ell 1 condition is useful when combined with the (Jet) Transversality Lemma in approximating a given holomorphic map by a holomorphic map transversal to a given submanifold. Condition Ell ${ }_{1}$ holds for holomorphic maps from any Stein manifold to any complex manifold $Y$ which enjoys the CAP property [11, Proposition 4.6 (b)]. In short the idea is to construct a finite collection of sprays on $Y$ using bundles described in Lemma 2.10 and combining them into map $H$ from the definition of $\mathrm{Ell}_{1}$. In particular, Ell ${ }_{1}$ holds for maps of Stein manifolds to manifolds of Class $\mathcal{A}$ since these enjoy the CAP property.

The following result from [11] follows from Lemma 2.5 and Sard's theorem. 
Lemma 2.7 ([11, Theorem 4.2]). Let $X$ be a Stein manifold and let $Y$ be a complex manifold such that holomorphic maps $X \rightarrow Y$ satisfy Condition Ell 1 . Choose a distance function $d$ on $Y$. Let $Z$ be a closed complex submanifold (or a closed complex subvariety) in $J^{k}(X, Y)$. Given a compact set $K \subset X$, a holomorphic map $f: X \rightarrow Y$ and an $\epsilon>0$, there is a holomorphic map $f_{1}: X \rightarrow Y$ such that

(1) $d\left(f(x), f_{1}(x)\right)<\epsilon$ for every $x \in K$, and

(2) $j^{k} f_{1} \pitchfork Z$ on $K$.

We will need the following lemma which was also used in the proof of Proposition 2 in $[6]$.

Lemma 2.8. The set $M^{r}(n, m)=\left\{A \in \mathbb{C}^{n \times m}: \operatorname{rank} A=r\right\}$ is a (nonclosed) complex submanifold of $\mathbb{C}^{n m}$ of complex codimension $(n-r)(m-r)$.

Proof. Let $A \in M^{r}(n, m)$. Change bases in $\mathbb{C}^{n}$ and $\mathbb{C}^{m}$ such that $A$ takes the form $A=\left[\begin{array}{ll}B & C \\ D & E\end{array}\right]$ where $B$ is an invertible $r \times r$ matrix. Denote by $U$ the neighborhood of $A$ in $\mathbb{C}^{n m}$ consisting of all matrices $A^{\prime}=\left[\begin{array}{ll}B^{\prime} & C^{\prime} \\ D^{\prime} & E^{\prime}\end{array}\right]$ where $B^{\prime}$ is invertible. Define a map $\Phi: U \rightarrow \mathbb{C}^{(n-r)(m-r)}$ by $\Phi\left(A^{\prime}\right)=E^{\prime}-D^{\prime} B^{\prime-1} C^{\prime}$. If $B^{\prime}, C^{\prime}, D^{\prime}$ are fixed, this is just a translation, and therefore $\Phi$ is a submersion.

To conclude the proof it now suffices to show $M^{r}(n, m) \cap U=\Phi^{-1}(0)$. Let $A^{\prime}=\left[\begin{array}{ll}B^{\prime} & C^{\prime} \\ D^{\prime} & E^{\prime}\end{array}\right] \in U$. Note that $F=\left[\begin{array}{cc}I_{r} & 0 \\ -D^{\prime} B^{\prime-1} & I_{n-r}\end{array}\right]$ is an invertible matrix and hence $\operatorname{rank} A^{\prime}=\operatorname{rank}\left(F A^{\prime}\right)$. But $F A^{\prime}=\left[\begin{array}{cc}B^{\prime} & C^{\prime} \\ 0 & E^{\prime}-D^{\prime} B^{\prime-1} C^{\prime}\end{array}\right]$ which has rank $r$ if and only if $E^{\prime}-D^{\prime} B^{\prime-1} C^{\prime}=0$, and this is equivalent to $A^{\prime} \in \Phi^{-1}(0)$.

Definition 2.9 (Spray on a manifold). A spray on a complex manifold $X$ is a holomorphic map $s: E \rightarrow X$ from total space of a holomorphic vector bundle $p: E \rightarrow X$ satisfying $s\left(0_{x}\right)=x$ for all $x \in X$. The spray is algebraic if $p: E \rightarrow X$ is an algebraic vector bundle and $s: E \rightarrow X$ is an algebraic map.

The following lemma is due to Gromov [13] (Lemmas 3.5B and 3.5C); see also [8, Lemma 1.3]. Here we supply additional details of the proof.

Lemma 2.10. Let $\widehat{Y}$ be an n-dimensional manifold of Class $\mathcal{A}_{0}$ and let $U \subset \widehat{Y}$ be a Zariski open subset biregularly isomorphic to $\mathbb{C}^{n}$ via an isomorphism $\varphi: U \rightarrow \mathbb{C}^{n}$. Let $\Lambda$ be a closed algebraic subset of $\widehat{Y}$ of pure dimension $n-1$ such that $U \cup \Lambda=\widehat{Y}$. Let $s: U \times \mathbb{C}^{n} \rightarrow U$ be a spray defined by

$$
s(y, t):=\varphi^{-1}(\varphi(y)+t), \quad y \in U, t \in \mathbb{C}^{n},
$$

and let $L=[\Lambda]^{-1}$ where $[\Lambda]$ is the line bundle defined by the divisor of $\Lambda$. There are an integer $m \in \mathbb{N}$ and an algebraic spray $\tilde{s}: E=\left(\widehat{Y} \times \mathbb{C}^{n}\right) \otimes L^{\otimes m} \rightarrow \widehat{Y}$ such that $\tilde{s}=s$ on $\left.E\right|_{\widehat{Y} \backslash \Lambda}$ and $s\left(E_{y}\right)=\{y\}$ for all $y \in \Lambda$. (Here we have identified $\left.E\right|_{\widehat{Y} \backslash \Lambda}$ with $(\widehat{Y} \backslash \Lambda) \times \mathbb{C}^{n}$ since $\left.L\right|_{\widehat{Y} \backslash \Lambda}$ is trivial. $)$

Proof. We can't just extend $s$ to $\widehat{Y} \times \mathbb{C}^{n}$ because of the singularities on $\Lambda$. However, we will show that any point $y \in \Lambda$ admits a Zariski neighborhood $\mathbb{C}^{n} \simeq V \subset \widehat{Y}$ such that $s$ extends to $\left.E\right|_{V}$ for $m \in N$ large enough. Since $\widehat{Y}=\bigcup_{j=1}^{r} U_{j}$ for Zariski 
open sets $U_{j}$ biregularly isomorphic to $\mathbb{C}^{n}$, with $U_{1}=U$, we will get the desired extension by choosing the largest $m$.

Let $\varphi_{j}: \mathbb{U}_{j} \rightarrow \mathbb{C}^{n}, 1 \leq j \leq r$, be biregular isomorphisms; the collection $\left\{\left(U_{j}, \varphi_{j}\right)\right.$ : $1 \leq j \leq r\}$ is then an algebraic atlas on $\widehat{Y}$. Choose $y_{0} \in \Lambda \backslash U$; without loss of generality we may assume that $y_{0} \in U_{2}$ and $\varphi_{2}\left(y_{0}\right)=0$. Recall that the spray $s$ is given in the local chart $U_{1} \times \mathbb{C}^{n}$ on $\widehat{Y} \times \mathbb{C}^{n}$ by $s_{1}^{\prime}(z, t)=z+t$. In the local chart $U_{2} \times \mathbb{C}^{n}$ the same spray is of the form

$$
s_{2}^{\prime}(z, t)=\varphi_{1,2}^{-1}\left(\varphi_{1,2}(z)+t\right), \quad z \in \varphi_{2}\left(U_{1,2}\right) \subset \mathbb{C}^{n}, t \in \mathbb{C}^{n},
$$

where $\varphi_{1,2}=\varphi_{1} \circ \varphi_{2}^{-1}$ and $U_{1,2}=U_{1} \cap U_{2}$. Clearly $s_{2}^{\prime}$ is holomorphic on the set

$$
\left\{(z, t) \in \mathbb{C}^{n} \times \mathbb{C}^{n}: z \in \varphi_{2}\left(U_{1,2}\right), \varphi_{1,2}(z)+t \in \varphi_{1}\left(U_{1,2}\right)\right\}
$$

and has singularities in the complement. In particular, $s_{2}^{\prime}$ is holomorphic at all points $(z, 0)$ with $z \in \varphi_{2}\left(U_{1,2}\right)$. Since $U_{2} \backslash U_{1} \subset \mathbb{P}_{n} \backslash U_{1} \subset \Lambda$, we have $\Omega:=$ $\mathbb{C}^{n} \backslash \varphi_{2}\left(\Lambda \cap U_{2}\right) \subset \varphi_{2}\left(U_{1,2}\right)$ and hence $s_{2}^{\prime}$ is holomorphic on a neighborhood of $\Omega \times\{0\}$.

For a fixed $z \in \Omega$ we can write $s_{2}^{\prime}(z, t)=z+\sum_{|\alpha|=1}^{\infty} f_{\alpha}(z) t^{\alpha}$, where $\alpha$ is a multiindex and $f_{\alpha}$ are matrices with rational functions as elements. Note that the transition maps of the bundle $E \rightarrow \widehat{Y}$ are $\Phi_{i j}: U_{i, j} \times \mathbb{C}^{n} \rightarrow U_{i, j} \times \mathbb{C}^{n}$ where

$$
\Phi_{i j}(y, t)=\left(y,\left(b_{j}(y) / b_{i}(y)\right)^{m} t\right) .
$$

Here $b_{j}$ is a regular defining function for $\Lambda \cap U_{j}$ and $b_{i}$ is a regular defining function for $\Lambda \cup U_{i}$. The bundle $\left.E\right|_{U_{1}}$ is trivial and can be identified with $U_{1} \times \mathbb{C}^{n}$. Denote by $\tilde{s}_{2}^{\prime}$ the map $s$ in the local chart $U_{2} \times \mathbb{C}^{n}$ on $E$. Then

$\tilde{s}_{2}^{\prime}(z, t)=\left(s_{1}^{\prime} \circ\left(\varphi_{1} \times \mathrm{id}\right) \circ \Phi_{12} \circ\left(\varphi_{2} \times \mathrm{id}\right)^{-1}\right)(z, t)=s_{2}^{\prime}\left(z, t b_{2}\left(\varphi_{2}^{-1}(z)\right)^{m} / b_{1}\left(\varphi_{2}^{-1}(z)\right)^{m}\right)$.

For $z \in U_{2} \cap \varphi_{2}\left(U_{1,2}\right)$ this can be written as

$$
\tilde{s}_{2}^{\prime}(z, t)=z+\sum_{|\alpha|=1}^{\infty} f_{\alpha}(z) \cdot t^{\alpha} b_{2}\left(\varphi_{2}^{-1}(z)\right)^{m|\alpha|} / b_{1}\left(\varphi_{2}^{-1}(z)\right)^{m|\alpha|}
$$

using the above series expansion for $s_{2}^{\prime}$. By the Cauchy formula for the coefficients of a power series for the rational map $s_{2}^{\prime}$, holomorphic on a neighborhood of $(z, 0)$, the maximum of the degrees of the poles of $f_{\alpha}(z)$ is bounded by some integer which is independent of $z$ and $\alpha$. Hence there is $m \in \mathbb{N}$ such that $b_{2}\left(\varphi_{2}^{-1}(z)\right)^{m} f_{\alpha}(z)$ is holomorphic on $\mathbb{C}^{n}$ and equals zero when $z \in \varphi_{2}\left(\Lambda \cap U_{2}\right),|\alpha| \in \mathbb{N}$. This shows that for such $m$ the map $\tilde{s}$ is holomorphic on $E$ at points $0 \in E_{y}, y \in \Lambda$. For other points $t \in \mathbb{C}^{n}$ we can still write $s_{2}^{\prime}$ as power series, since the intersection of the singular set of $s_{2}^{\prime}$ with $\{z\} \times \mathbb{C}^{n} \equiv \mathbb{C}^{n}$ is nowhere dense in $\mathbb{C}^{n}$. Furthermore, because of the factors $b_{2}\left(\varphi_{2}^{-1}(z)\right)^{m}$ we can extend $\tilde{s}_{2}^{\prime}$ to a continuous, locally bounded map on a neighborhood of hypersurfaces $\varphi_{2}\left(\Lambda \cap U_{2}\right) \times \mathbb{C}^{n}$ with $\tilde{s}_{2}^{\prime}(z, t)=z$ for $z \in \varphi_{2}\left(\Lambda \cap U_{2}\right)$, $t \in \mathbb{C}^{n}$. By the Riemann extension theorem $\tilde{s}_{2}^{\prime}$ extends to a holomorphic map on a neighborhood of $\varphi_{2}\left(\Lambda \cap U_{2}\right) \times \mathbb{C}^{n}$.

\section{Proofs of MAIN THEOREMS}

The following is Lemma 3.4 in $[9$, p. 156] with $r=n-2, s=2$ and $D \times L$ instead of $L$. 
Lemma 3.1. Let $K \subset \mathbb{C}^{n}$ be a product of a closed polydisc and a ball, and let $\Sigma \subset \mathbb{C}^{n} \backslash K$ be an algebraic set with $\operatorname{dim} \Sigma \leq n-2$. Let $D=\pi(K)$, where $\pi: \mathbb{C}^{n-2} \times$ $\mathbb{C}^{2} \rightarrow \mathbb{C}^{n-2}$ is a standard projection and $L \subset \mathbb{C}^{2}$ is a compact polydisc such that $K \subset D \times L$. Given $\epsilon>0$ there exists an automorphism $\Psi$ of $\mathbb{C}^{n}$ of the form $\Psi\left(z^{\prime}, z^{\prime \prime}\right)=\left(z^{\prime}, \psi\left(z^{\prime}, z^{\prime \prime}\right)\right)\left(z^{\prime} \in \mathbb{C}^{n-2}, z^{\prime \prime} \in \mathbb{C}^{2}\right)$ such that

(i) $|\Psi(z)-z|<\epsilon$ for all $z \in K$, and

(ii) $\Psi(D \times L) \subset \mathbb{C}^{n} \backslash \Sigma$.

The following lemma is the main ingredient in the proof of Theorem 1.1.

Lemma 3.2. Let $Y$ be a manifold of class $\mathcal{A}$ with $\operatorname{dim} Y=p$. Choose a distance function d on $Y$. Let $K \subset \mathbb{C}^{n}=\mathbb{C}^{n-2} \times \mathbb{C}^{2}, L \subset \mathbb{C}^{2}, D \subset \mathbb{C}^{n-2}$ be as in Lemma 3.1. Let $r \in \mathbb{N}$ satisfy $(n-r+1)(p-r+1) \geq 2$. Given a holomorphic map $f: K \rightarrow Y$ satisfying rank $f \geq r$ on $K$ and an $\epsilon>0$, there exists an algebraic map $\tilde{f}: D \times L \rightarrow Y$ such that

(i) $d(\tilde{f}(z), f(z))<\epsilon$ for all $z \in K$, and

(ii) $\operatorname{rank} \tilde{f} \geq r$ on $D \times L$.

Proof. By Corollary 3.2 in [11] we can approximate the map $f: K \rightarrow Y$ with an algebraic map $\mathbb{C}^{n} \rightarrow Y$. So we can assume that $f$ is algebraic, defined on the whole $\mathbb{C}^{n}$ and with rank $f \geq r$ on $K$. Let $\Sigma_{f, r}=\left\{z \in \mathbb{C}^{n}: \operatorname{rank}_{z} f<r\right\}$. Then $\Sigma_{f, r} \cap K=\emptyset$ provided the above approximation was good enough.

If $\operatorname{dim} \Sigma_{f, r} \leq n-2$, Lemma 3.1 furnishes an automorphism $\Psi$ of $\mathbb{C}^{n}$ which approximates the identity on $K$ and satisfies $\Psi(D \times L) \subset \mathbb{C}^{n} \backslash \Sigma_{f, r}$. The map we are looking for is $\tilde{f}=f \circ \Psi$.

Now suppose that $\operatorname{dim} \Sigma_{f, r}=n-1$. We will reduce this to the previous case $\operatorname{dim} \Sigma_{f}=n-2$. This reduction is similiar to the one used in the proof of Proposition 5.4 in [11]. By the definition of a Class $\mathcal{A}$ manifold we have $Y=\widehat{Y} \backslash A$ where $\widehat{Y}$ is a manifold of Class $\mathcal{A}_{0}$ and $A$ an algebraic subset of codimension at least two in $\widehat{Y}$. We will approximate $f$ on $D \times L$ by an algebraic map $f_{0}: \mathbb{C}^{n} \rightarrow \widehat{Y}$ such that $\operatorname{dim} \Sigma_{f_{0}, r} \leq n-2$. By approximating well enough we will also get $f_{0}(D \times L) \subset \widehat{Y} \backslash A$. In each step of the approximation a given map $f$ will be replaced by a nearby algebraic map $f_{1}$ such that the corresponding set $\Sigma_{f_{1}, r} \subset \mathbb{C}^{n}$ has fewer $n-1$ dimensional irreducible components than $\Sigma_{f}$.

Choose a point $z_{0} \in \Sigma_{f}$ belonging to exactly one $(n-1)$-dimensional irreducible component $\Sigma^{\prime}$ of $\Sigma_{f, r}$. Set $y_{0}=f\left(z_{0}\right)$. By the definition of a class $\mathcal{A}$ manifold there is a Zariski open neigborhood $U$ of $y_{0}$ in $\widehat{Y}$ which is biregularly isomorphic to $\mathbb{C}^{p}$, where $p=\operatorname{dim} \widehat{Y}$. Hence there is a biregular isomorphism $\varphi: U=\widehat{Y} \backslash \Lambda \rightarrow$ $\mathbb{C}^{n}=\mathbb{P}_{n} \backslash H$ where $H$ is the plane at infinity in $\mathbb{P}_{n}$. Since $\varphi$ has poles at $\Lambda$ it can be viewed as a holomorphic map $\widehat{Y} \rightarrow \mathbb{P}_{n}$. Let $\varphi\left(z_{0}\right) \in V \subset \mathbb{P}_{n}$ where $V \equiv \mathbb{C}^{n}$. There is a polynomial $q$ defined on $V$ which vanishes on $\varphi(\Lambda) \cap V$ but $q\left(\varphi\left(y_{0}\right)\right) \neq 0$. The closure of the zero locus of $q$ in $\mathbb{P}_{n}$ is an algebraic set. Denote by $\widehat{\Lambda}$ its $\varphi$-preimage. Then $\widehat{\Lambda}$ is an algebraic set in $\widehat{Y}$ of pure dimension $p-1$ such that $\widehat{\Lambda} \cup U=\widehat{Y}$ and $y_{0} \notin \widehat{\Lambda}$.

Let $L$ be a holomorphic line bundle over $\widehat{Y}$ defined by the divisor of $\widehat{\Lambda}$. Using Lemma 2.10 we get a spray $s: E=\left(\tilde{Y} \times \mathbb{C}^{p}\right) \otimes L^{-m} \rightarrow \widehat{Y}$ such that $s(x, t)=$ $x+t$ on $U$ (identifying $\left.E\right|_{U}$ with $U \times \mathbb{C}^{p}$ and $U$ with $\mathbb{C}^{p}$ via an isomorphism) and $s(x, t)=x$ for all $x \in \widehat{\Lambda}$. Let $\iota: f^{*} E \rightarrow E$ be a natural map covering $f$. Here $f^{*} E=\left\{(z, v): z \in \mathbb{C}^{n}, v \in E_{f(z)}\right\}$ is the pullback of the bundle $p: E \rightarrow \widehat{Y}$. In local 
coordinates $\iota$ is just $\iota(z, v)=(f(z), v)$. Since $f$ is algebraic, $f^{*} E$ is an algebraic vector bundle over $\mathbb{C}^{n}$. By Serre's theorem A [21] the bundle $f^{*} E$ is generated by finitely many algebraic sections, and hence there is a surjective algebraic vector bundle map $g: \mathbb{C}^{n} \times \mathbb{C}^{q} \rightarrow f^{*} E$ for some $q \in \mathbb{N}$. We can write $g(z, t)=\sum_{j=1}^{q} g_{j}(z) t_{j}$ where $g_{j}: \mathbb{C}^{n} \rightarrow f^{*} E$ are sections. Set $\Phi=s \circ \iota \circ g, \Lambda=f^{-1}(\widehat{\Lambda}), V=\mathbb{C}^{n} \backslash \Lambda$. From the above statements it is easy to conclude the following properties of the algebraic bundle map $H: \mathbb{C}^{n} \times \mathbb{C}^{q} \rightarrow \widehat{Y}$ :

- $H(z, 0)=f(z)$ for $z \in \mathbb{C}^{n}$,

- $H(z, t)=z$ for $z \in \Lambda$ and $t \in \mathbb{C}^{q}$, and

- $H(z, \cdot)$ is a submersion on $\mathbb{C}^{q}$ for every $z \in V$.

Let $W$ denote the space of all quadratic polynomial maps $\mathbb{C}^{n} \rightarrow \mathbb{C}^{q}$. Set $f_{P}(z)=$ $H(z, P(z))$ for $P \in W$. By Lemma 2.8 the set $Z_{j}=\left\{(z, y, \alpha) \in J^{1}\left(\mathbb{C}^{n}, \widehat{Y}\right): \operatorname{rank} \alpha=\right.$ $j$ \} is a submanifold of $J^{1}\left(\mathbb{C}^{n}, \widehat{Y}\right)$ of codimension $(n-j)(p-j)$ and $Z=\bigcup_{j=0}^{r-1} Z_{j}$ is a closed subvariety of codimension $(n-r+1)(p-r+1) \geq 2$. By Lemma 2.5 for every $P$ in some open dense subset of $W$ we get $j^{1} f_{P} \pitchfork Z$. If we choose $P$ close to 0 , from a subset of polynomials with up to first degree terms equal to zero, we can conclude the following about the map $f_{P}: \mathbb{C}^{n} \rightarrow \widehat{Y}$ :

(1) $f_{P}(z)=f(z), d f_{P}(z)=d f(z)$ for $z \in \Lambda$,

(2) $f_{P}$ approximates $f$ on $D \times L$, and

(3) the algebraic set $\Sigma_{f_{P}, r}=\left(j^{1} f_{P}\right)^{-1}(Z)$ has dimension less than $n-1$ at every point of $V$.

By (3) the only remaining irreducible $n$-1-dimensional components of $f_{P}$ are those lying in $\Lambda$, where they are equal to those of $\Sigma_{f, r} \cap \Lambda$. Hence the number of $n$-1-dimensional components intersecting with $\Lambda$ has not increased. At least one component $\Sigma^{\prime}$ from $\Sigma_{f, r}$ is missing since $z_{0} \notin \Lambda$. Set $\Sigma_{1}=\Sigma_{f_{P}, r}, f_{1}=f_{P}$. The map $f_{1}: \mathbb{C}^{n} \backslash \Sigma_{1} \rightarrow \widehat{Y}$ has rank $\geq r$ and $\Sigma_{1}$ has fewer $(n-1)$-dimensional components than $\Sigma_{f, r}$. By repeating this procedure we obtain in finitely many steps the desired algebraic map $f_{0}$ with $\operatorname{dim} \Sigma_{f_{0}} \leq n-2$.

Lemma 3.3. Let $Y$ be a manifold of class $\mathcal{A}$ with $\operatorname{dim} Y=p$. Let $K \subset \mathbb{C}^{n}$ be a product of a closed ball and a closed polydisc, and let $Q \subset \mathbb{C}^{n}$ be a closed polydisc containing $K$. Let $r$ be such that $(n-r+1)(p-r+1) \geq 2$. Every holomorphic map $f: K \rightarrow Y$ with rank $f \geq r$ on $K$ can be approximated uniformly on $K$ by algebraic maps $\tilde{f}: Q \rightarrow Y$ satisfying $\operatorname{rank} \tilde{f} \geq r$ on $Q$.

Proof. If $n$ is even, we write $\mathbb{C}^{n}=\mathbb{C}^{2} \times \cdots \times \mathbb{C}^{2}(n / 2$ factors $)$ and let $\pi_{j}: \mathbb{C}^{n} \rightarrow$ $\mathbb{C}^{n-2}$ be the projection whose kernel is the $j$-th factor. Let $Q=L_{1} \times \cdots \times L_{m}$ where $L_{j} \subset \mathbb{C}^{2}$ are polydiscs. Using Lemma 3.2 we approximate $f=f_{0}$ on $K=$ $K_{0}$ by $f_{1}: L_{1} \times \pi_{1}(K) \rightarrow Y$. Set $K_{1}=L_{1} \times \pi_{1}(K)$ and approximate $f_{1}$ on $K_{1}$ by $f_{2}: L_{2} \times \pi_{2}\left(K_{1}\right) \rightarrow Y$ using Lemma 3.2 (for purposes of shorter notation the coordinates have been permuted). By continuing in this fashion we get the desired approximation in $m=n / 2$ steps. In the case of odd $n$ we use an extra disc.

Proof of Theorem 1.1. By the definition of a class $\mathcal{A}$ manifold we have $Y=\widehat{Y} \backslash A$, where $\widehat{Y}$ is a manifold of Class $\mathcal{A}_{0}$ and $A$ is a closed algebraic subset of $\widehat{Y}$ of codimension at least two. Choose a distance function $d$ on $\widehat{Y}$ induced by a complete Riemannian metric. 
Suppose that $K$ is a closed polydisc in $\mathbb{C}^{n}$. Choose an exhaustion of $\mathbb{C}^{n}$ with closed polydiscs $Q_{j}, j \in \mathbb{Z}_{+}$, where $Q_{0}=K \subset Q_{1}$.

By Corollary 3.2 in [11] we can approximate $f$ uniformly on $K$ by an algebraic map $f_{0}: \mathbb{C}^{n} \rightarrow Y$.

By Lemma 2.7 we can approximate $f_{0}$ uniformly on $Q_{0}$ by a holomorphic map transversal to $A$ on $Q_{0}$, and hence we can assume that $f_{0} \pitchfork A$ on $Q_{0}$. Choose a positive number $\delta_{0}>0$ such that every holomorphic map $g: \mathbb{C}^{n} \rightarrow \widehat{Y}$ with $d\left(g(z), f_{0}(z)\right)<\delta_{0}$ for all $z \in Q_{1}$ satisfies rank $g \geq r$ on $Q_{0}$ and $g \pitchfork A$ on $Q_{0}$. Using Lemma 3.3 and Corollary 3.2 in [11] we get a holomorphic map $f_{1}: \mathbb{C}^{n} \rightarrow Y$ satisying $\operatorname{rank} f_{1} \geq r$ on $Q_{1}$ and $d\left(f_{1}(z), f_{0}(z)\right)<\min \left(\epsilon / 2, \delta_{0} / 2\right)$ for all $z \in Q_{0}$.

Proceeding inductively we get a sequence of holomorphic maps $f_{j}: \mathbb{C}^{n} \rightarrow Y$ and a decreasing sequence of positive numbers $\delta_{j}>0$ satisfying the following:

(i) $d\left(f_{j+1}(z), f_{j}(z)\right)<\min \left(\epsilon / 2^{j+1}, \delta_{j} / 2\right)$ for all $z \in Q_{j}$ and $j \geq 0$,

(ii) rank $f_{j} \geq r$ on $Q_{j}$ and $f_{j} \pitchfork A$ on $Q_{j}$, and

(iii) every holomorphic map $g: \mathbb{C}^{n} \rightarrow \widehat{Y}$ with $d\left(g(z), f_{j}(z)\right)<\delta_{j}$ for all $z \in Q_{j+1}$ satisfies rank $g \geq r$ on $Q_{j}$ and $g \pitchfork A$ on $Q_{j}$.

The sequence of holomorphic maps $f_{j}$ converges uniformly on the compacts in $\mathbb{C}^{n}$ to a holomorphic map $F: \mathbb{C}^{n} \rightarrow \widehat{Y}$ satisfying $d(F(z), f(z))<\epsilon$ for all $z \in Q_{0}=K$ (a consequence of (i)) and $d\left(F(z), f_{j}(z)\right)<\delta_{j}$ for all $z \in Q_{j}$ and $j \geq 0$ (because of (ii) and the definition of the numbers $\delta_{j}$ ).

This implies $F \pitchfork A$ on $\mathbb{C}^{n}$ and $\operatorname{rank} F \geq r$ on $\mathbb{C}^{n}$. To show that $F\left(\mathbb{C}^{n}\right) \subset Y=$ $\widehat{Y} \backslash A$ suppose $F(z) \in A$ for some $z \in \mathbb{C}^{n}$. The transversality condition $F \pitchfork A$ implies $\operatorname{rank}_{z} F+\operatorname{dim}_{z} A \geq p=\operatorname{dim} \widehat{Y}$. This and $F \pitchfork_{z} A$ implies $g(U) \cap A \neq \emptyset$ for all holomorphic maps $g: U \rightarrow \widehat{Y}$ close enough to $F$ on some neighborhood $U$ of $z$ in $\mathbb{C}^{n}$. Since $f_{j}: \mathbb{C}^{n} \rightarrow Y$ for all $j \geq 0$, we have a contradiction. This completes the proof of Theorem 1.1.

Proof of Theorem 1.6. Case (1): Let $Z=\bigcup_{j=0}^{r-1} Z_{j}$ where $Z_{j}$ is the submanifold of $J^{1}(X, Y)$ consisting of all jets with rank $j$. Now $\Sigma_{f, r}=\left(j^{1} f\right)^{-1}(Z)$. Since the codimension of $Z$ is $(n-r+1)(p-r+1)$ (see Lemma 2.8), we will get $\operatorname{dim} \Sigma_{f, r}=$ $n-(n-r+1)(p-r+1)$ if $j^{1} f \pitchfork Z$. If this holds, then the set $\left\{f \in \mathcal{H}(X, Y): j^{1} f \pitchfork\right.$ $Z\} \subset \Omega$ will satisfy the conclusion of Theorem 1.6.

Choose an exhaustion of $X$ by compact sets $K_{l}, l \in \mathbb{N}$, and let

$$
\Omega_{l}=\left\{f \in \mathcal{H}(X, Y): j^{1} f \pitchfork Z \text { on } K_{l}\right\} .
$$

Then $\Omega=\bigcap_{l \in \mathbb{N}} \Omega_{l}$. Since $\mathcal{H}(X, Y)$ is a Baire space, it is enough to show that $\Omega_{l}$ is open and dense in $\mathcal{H}(X, Y)$. Openness follows directly from the definition of transversality and the fact that $Z$ is closed (if we perturb $f$ on neighborhood of $K$ a little, the transversality condition will still be satisfied on $K$ by the Cauchy inequality for derivatives). To prove density choose $g \in \mathcal{H}(X, Y)$ and a compact subset $L \subset X$. Using Lemma 2.7 we get a holomorphic map $f$ which approximates $g$ on $L \cup K_{l}$ and satisfies the transversality condition in the definition of $\Omega_{l}$.

Case (2): The assumed inequality implies $\operatorname{dim} X+\operatorname{dim} Z<\operatorname{dim} J^{1}(X, Y)$. Therefore $j^{1} f \pitchfork Z$ implies $j^{1} f(X) \cap Z=\emptyset$ which is equivalent to rank $f \geq r$ on $X$. The set of such $f$ is open and dense by the proof of case (1).

\section{ACKNOWLEDGMENTS}

I wish to thank F. Forstnerič for many useful suggestions. 


\section{REFERENCES}

[1] R. Abraham, Transversality in manifolds of mappings, Bull. Amer. Math. Soc. 69 (1963), 470-474. MR0149495 (26:6982)

[2] W. Barth, K. Hulek, C. A. M. Peters and A. van de Ven, Compact Complex Surfaces, 2nd ed., Springer-Verlag, Berlin, 2004. MR2030225 (2004m:14070)

[3] E. M. Chirka, Complex Analytic Sets, Kluwer Academic Publishers, Dordrecht, 1989. MR1111477 (92b:32016)

[4] J.-P. Demailly and J. El Goul, Hyperbolicity of generic surfaces of high degrees in projective 3-space, Amer. J. Math. 122 (2000), 515-546. MR1759887 (2001f:32045)

[5] D. A. Eisenman, Intrinsic measures on complex manifolds and holomorphic mappings. Memoirs of the Amer. Math. Soc. 96, American Mathematical Society, Providence, Rhode Island, 1970. MR0259165 (41:3807)

[6] O. Forster, Plongements des variétés de Stein, Comment. Math. Helv. 45(1970), 170-184. MR0269880 (42:4773)

[7] F. Forstnerič, Interpolation by holomorphic automorphisms and embeddings in $\mathbb{C}^{n}$, J. Geom. Anal. 9 (1999), 93-118. MR1760722 (2001g:32040)

[8] , The Oka principle for sections of subelliptic submersions, Math. Z. 241 (2002), 527-551. MR1938703 (2003i:32043)

[9] Noncritical holomorphic functions on Stein manifolds, Acta Math., 191 (2003), 143189. MR2051397 (2005b:32021)

[10] , Runge approximation on convex sets implies Oka's property. Annals of Math. 163 (2006), 689-707. MR2199229 (2006j:32011)

[11] Holomorphic flexibility properties of complex manifolds, Amer. J. Math. 128 (2006), 239-270. MR2197073 (2006k:32024)

[12] P. Griffiths, J. Harris, Principles of algebraic geometry. Wiley Classics Library. John Wiley \& Sons, Inc., New York, 1994. MR1288523 (95d:14001)

[13] M.Gromov, Oka's principle for holomorphic sections of elliptic bundles, J. Amer. Math. Soc. 2 (1989), 851-897. MR1001851 (90g:32017)

[14] — Partial differential relations, Ergeb. Math. Grenzgeb. (3), 9, Springer-Verlag, Berlin, 1986.

[15] R. C. Gunning, H. Rossi, Analytic functions of several complex variables, Prentice-Hall, Englewood Cliffs, 1965. MR0180696 (31:4927)

[16] S. Kaliman and F. Kutzschebauch, Criteria for the density property of complex manifolds, Invent. Math. (to appear).

[17] S. Kaliman and M. Zaidenberg, A transversality theorem for holomorphic mappings and stability of Eisenman-Kobayashi measures, Trans. Amer. Math. Soc 348 (1996), 661-672. MR1321580 (96g:32043)

[18] S. Kobayashi, Hyperbolic Manifolds and Holomorphic Mappings, Marcel Dekker, New York, 1970. MR0277770 (43:3503)

[19] K. Kodaira, Holomorphic mappings of polydiscs into compact complex manifolds. J. Diff. Geom. 6 (1971/72), 33-46. MR0301228 (46:386)

[20] J.-P. Rosay and W. Rudin, Holomorphic maps from $\mathbb{C}^{n}$ to $\mathbb{C}^{n}$, Trans. Amer. Math. Soc. 310 (1988), 47-86. MR929658 (89d:32058)

[21] J.-P. Serre, Faisceaux algébriques cohérents, Ann. of Math. 61 (1955), 197-278. MR0068874 (16:953c)

[22] G. Stolzenberg, Polynomially and rationally convex sets, Acta Math. 109 (1963), 259-289. MR0146407 (26:3929)

[23] V. Guillemin and A. Pollack, Differential Topology, Prentice-Hall, Englewood Cliffs, 1974. MR0348781 (50:1276)

Institute of Mathematics, Physics and Mechanics, University of Ljubljana, JadranSKA 19, 1000 Ljubluana, Slovenia

E-mail address: dejan.kolaric@fmf.uni-lj.si 